\title{
ANÁLISIS DE LOS NIVELES DE ACTIVIDAD FÍSICA EN UN GRUPO DE ADULTOS MAYORES DURANTE LA CUARENTA COVID-19 EN BOGOTÁ
}

Physical activity levels analysis in old age group during the Covid-19 quarantine in Bogotá

\author{
BRAYAN DAVID OCHOA PÁEZ \\ bochoa3@estudiantes.areandina.edu.co \\ JUAN PABLO ÁNGEL RODRÍGUEZ \\ CATALINA GUTIÉRREZ GÓMEZ
}

Fundación Universitaria del Área Andina (Colombia)

Artículo de investigación formativa

Recepción: 20 de noviembre de 2020

Aceptación: 10 de marzo de 2021 


\section{Resumen:}

Este estudio analizó los niveles de actividad física de adultos mayores institucionalizados de la localidad de Barrios Unidos. Es una investigación cuantitativa, de tipo descriptivo y corte transversal. Para el proceso se contemplaron los procedimientos éticos emitidos en la Declaración de Helsinki y la Resolución 8430 de 1993, expedida por el Ministerio de Salud de Colombia; así como el aval de Comité de Ética de la Fundación Universitaria del Área Andina. Los 54 adultos mayores están vinculados a un hogar geriátrico en esta localidad de Bogotá, quienes presentan su participación voluntaria en el proceso por medio del consentimiento informado. Se generó la aplicación del International Physical Activity Questionary (IPAQ) para analizar los niveles de actividad física durante proceso de aislamiento obligatorio durante la pandemia de COVID 19 en Colombia. Además, desarrolló la valoración por medio de verificación electrónica, generando la aclaración de dudas de forma remota y ayudados por los cuidadores. Los adultos mayores generan actividades físicas orientadas y dirigidas con un nivel medio, de acuerdo con los estándares internacionales. Sin embargo, entre mayor longevidad se observa un mayor estado de quietud que aumenta los riesgos para su salud. La actividad física ha sido reconocida por sus múltiples factores de beneficio a la salud física y mental, especialmente para los adultos mayores quienes, debido a su proceso natural de envejecimiento, son más propensos a desarrollar diferentes patologías como disminución de la calidad muscular, aumento del tejido adiposo o riesgo de enfermedades no transmisibles.

Palabras clave: adulto mayor; actividad física; Covid-19.

\section{Abstract:}

This paper analyzes the physical activity levels from old age group institutionalized in Barrios Unidos locality. It is a quantitative research, from a descriptive type and cross section. To the process, it contemplates the ethical procedures issued by Helsinki declaration and the 8430 resolution from 1993, by the Colombian health ministry; as well as the endorsement from the ethical committee in Fundación Universitaria del Área Andina. The 54 elderly people are linked with a geriatric home in this locality, in Bogotá. They present their volunteer participation in the process through informed consent. It generates the International Physical Activity Questionary (IPAQ) application to analyze the physical activities levels during the obligatory isolation process in COVID 19 pandemic in Colombia. Besides, it develops an assessment via an electronic verification; generating the doubts clarification in a remote way and helped by the caretakers. The elderly people generate physical activities orientated and directed with a high level, in accordance with international standards. However, the longer the longevity, it identifies 
a major stillness state that increase the health risks. The physical activity has been recognizing by its multiples benefit factors in physical and mental health, especially for elderly people whose natural aging process developed different pathologies as the decrease of muscle quality, increased adipose tissue or non-communicable diseases risks.

Keywords: old age; physical activity; Covid-19

\section{Introducción}

El envejecimiento es un proceso natural y universal, pero a la par complejo. Allí interactúan variables como la genética, el medio ambiente, el estilo de vida y las enfermedades crónicas entre otras. Del modo en cómo lo hagan va a depender en gran medida la forma de envejecer. La vejez está asociada a una pérdida de masa muscular, denominada sarcopenia. Se inicia en la cuarta década de la vida con una disminución de fuerza de alrededor del uno (1) por ciento al año y se acelera con el transcurso del tiempo (Cruz-Jentoft et al., 2010).

Para la Organización Panamericana de la Salud (OPS, 2011), un adulto mayor es aquel individuo que se encuentra en la última etapa de la vida, lo que sigue tras la etapa de adultez; antecede al fallecimiento de la persona ya que durante esta fase el cuerpo y las facultades cognitivas de las personas se van deteriorando. Ante estos diversos cambios, se caracteriza porque el organismo representa una serie de cambios fisiológicos asociados a su metabolismo, desarrollo cognitivo, motriz y de desempeño en la mayoría de los sistemas y funciones corporales (Castellanos-Ruiz, Gómez-Gómez y Guerrero-Mendieta, 2017).

En el proceso de envejecimiento son dos (2) factores los que deben considerase por su relevancia: los biológicos y los físicos. Producen cambios importantes en las células, tejidos, órganos y sistemas dentro del organismo. Esto se acentúa cuando se ven alteradas ciertas funciones físicas, como la pérdida de movilidad, fuerza en los músculos; además de presentar fracturas y patologías que impiden el funcionamiento físico del adulto mayor (Rodrigues, Hauser, Capeletto, Petreça, Faleiro y Mazo, 2019).

De acuerdo con Gómez (2012), en su estudio determina que aparecen una serie de cambios en la composición corporal, con un aumento y redistribución de la masa grasa y un descenso de la masa magra. Por ello, la pérdida de masa y función muscular está asociada a la edad y representa la sarcopenia; un síndrome caracterizado por el desgaste generalizado y progresivo del músculo esquelético. En el sistema cardiovascular son característicos el aumento de la rigidez arterial y del grosor de la pared ventricular, así como la disminución de la contractilidad del miocardio. 
De este modo, los cambios del estado de la actividad física pueden llegar a desarrolla la aparición de la sarcopenia. Contribuye a una disminución de la capacidad funcional e independencia en las actividades de la vida diaria y afecta a la calidad de vida de las personas mayores.

Dicho proceso incluye la pérdida de masa muscular, de fuerza y cambios cualitativos en el tejido muscular (Vagetti, Oliveira, Pereira, Pacífico, Alves y Campos, 2017). Se considera que están implicados en dicha pérdida una amplia variedad de procesos como la alteración en la síntesis y degradación de proteínas, la inflamación, las alteraciones hormonales y la disfunción mitocondrial (Cruz-Jentoft et al., 2010).

La actividad física es uno de los elementos que permite evitar la prevalencia de riesgo al desarrollo de la sarcopenia. Se puede evidenciar que la práctica de una actividad física es considerada el arma secreta para sentirse mejor, vivir más tiempo y desacelerar el proceso de envejecimiento. En el adulto mayor es normal que se presenten cambios a nivel físico como lo mencionan Castellanos, Gómez y Guerrero (2017).

Otros factores externos a la edad influyen en la pérdida de la masa muscular, el más relevante es el sedentarismo. Por eso es tan importante que se adquieran hábitos de vida que permitan desarrollar acciones de movimiento en edades más longevas, para permitir que los adultos se mantengan activos. Los procesos de adquisición para realizar programas de actividad física, pueden relacionar ejercicios asociados al entrenamiento de la fuerza, que puede contribuir no solo a tonificar la masa muscular, mejorar el desarrollo muscular; siempre y cuando esté bien planeado el proceso de trabajo y se acompañe con una alimentación adecuada.

Un mayor desarrollo de masa muscular en los adultos mayores contribuye a la prevención de patologías óseas, consiguiendo una mayor densidad ósea. De esa forma, se genera mayores beneficios en las mujeres, donde se puede prevenir la aparición de la osteoporosis. Asimismo, este tipo de actividades aumenta la coordinación y el control corporal; puede ayudar a los mejorar la estabilidad y evitar el riesgo de caídas. Es un factor de riesgo a desarrollar lesiones osteomusculares de tipo traumático (Padilla, Sánchez y Cuevas, 2014).

El deterioro con la edad en la función muscular es uno de los principales factores que influyen en la disminución de la capacidad de vida independiente de las personas. Con el envejecimiento parece que se observa una relación curvilínea entre la edad y la fuerza muscular (Alonso e Izquierdo, 2003). Diversos estudios han mostrado que la realización de un entrenamiento sistemático de fuerza máxima se acompaña de incrementos significativos en la producción de fuerza independientemente de la edad y el sexo, siempre y cuando la intensidad y duración del periodo de entrenamiento sean suficientes (Alonso e Izquierdo, 2003). 
La mayoría de los autores señalan que al llegar a la frontera de los 60 años comienza una etapa caracterizada por la reducción gradual de la fuerza máxima. Se ha estimado que la pérdida de fuerza puede ser de un 12 a 15\% por década. De tal manera, la disminución de la fuerza suele ser del orden de 30 al 40\%, cuando se llega a los 70 años de edad. La disminución de la fuerza permanece constante hasta la octava década de vida; a partir de esta edad se produce una mayor aceleración en la disminución

Otras de las propiedades otorgadas a la práctica regular de actividad física se relacionan con la prevención de diabetes, enfermedades cardiovasculares; así como puede ser parte del tratamiento de artritis, ansiedad y depresión, que comúnmente son problemas de salud que se presentan en los adultos mayores. El ejercicio mejora la calidad de vida de esta población y les permite ser más independiente a las actividades recreativas y de desplazamientos fuera de su consumo energético de reposo, como excursiones caminando o en bicicleta, tareas domésticas como la jardinería, juegos, deportes o ejercicios programados.

\section{Metodología}

\section{Tipo de estudio.}

El estudio es de tipo cuantitativo descriptivo, de corte transversal y aporta a una variable de un estudio institucional (Daza-Orozco y Cera-Ochoa, 2018); generado por el programa profesional en entrenamiento deportivo; está orientado a verificar el estado de salud física y desarrollo motor en adultos mayores. En una población de adultos mayores institucionalizados se aplican diferentes cuestionarios para determinar el estado de actividad física de la población, por medio de instrumentos validados como el International Physical Questionary Short (IPAQ). Alli se generó un auto reporte emitido por los adultos mayores o por sus cuidadores, respondiendo las siete (7) preguntas del instrumento.

\section{Muestra.}

Se representó una evaluación con 55 adultos mayores de género masculino de un hogar geriátrico perteneciente a la localidad de Barrios Unidos de Bogotá. Estos adultos mayores aceptaron de forma voluntaria participar en el estudio, por medio de un consentimiento informado que está avalado por el Comité de Ética de la Fundación Universitaria del Área Andina.

El documento se generó por recursos electrónicos, emitidos mediante correo electrónico y ejecutados por parte de los cuidadores. Ellos fueron instruidos en el instrumento, mediante acompañamiento de los investigadores principales.

El documento explica las características de las variables, buscando analizar el estado de actividad física en los últimos siete (7) días. Así, genera reserva de 
la identidad del evaluado; cumpliendo con los criterios emitidos en la Declaración del Helsinki y la Resolución 1350 del Ministerio de Salud de Colombia, indicando que es una investigación de riesgo bajo.

\section{Criterios de inclusión y exclusión.}

Para este proceso se tuvo en cuenta los siguientes criterios de selección y exclusión para el proceso:

1.Participar voluntaria por parte de los sujetos o por su tutor o la persona legalmente responsable del sujeto.

2.Ser una persona mayor de 60 años, con capacidad de movilizarse con ayuda parcial (bastón).

3.Identificar el consumo de medicamentos que generen alteraciones como beta bloqueadores, inmunodepresores y otro tipo de medicamentos.

4.No presentar lesión osteomuscular que generen limitación de movimiento.

\section{Procedimiento.}

Se emite invitación de participación al hogar geriátrico quien genera la participación de este proceso. Se realiza reunión por video llamada en donde se describe el proceso por el cual se desea aplicar el cuestionario para la toma de los datos de los adultos mayores. Así, se emite el documento por correo y este es expuesto por parte de los investigadores, resolviendo dudas por parte de los adultos mayores. Ellos contaron con 14 días calendario para responderlo y expresar sus inquietudes.

\section{IPAQ.}

El International Physical Activity Questionary (IPAQ), empleado en este proceso corresponde al modelo validado para Colombia. Se puede aplicar a personas de 18 a 65 años de edad para determinar el tiempo que se emplea para la actividad física por semana y proporciona datos por medio de siete (7) preguntas que abarcan el tiempo que dedica tiempo libre, al mantenimiento del hogar y el tiempo que realiza la actividad física. Este cuestionario emite preguntas, en su totalidad fueron respondidas con horas y minutos de dedicación a las actividades descritas:

- ¿Cuánto actividades físicas intensas realizó tales como levantar pesos pesados, cavar, ejercicios aeróbicos o andar rápido en bicicleta?

- ¿Cuántos días hizo actividades físicas moderadas tales como transportar pesos livianos o andar en bicicleta a velocidad regular?

- ¿Cuánto tiempo en total dedicó a una actividad física moderada en uno de esos días?

- ¿Cuánto tiempo en total dedicó a una actividad física intensa en uno de estos días? 
•¿Cuántos días caminó por lo menos diez (10) minutos seguidos?

• ¿Cuánto tiempo en total dedicó a caminar en uno de esos días?

•¿Cuánto tiempo paso sentado durante un día hábil?

Estas preguntas se realizan teniendo en cuenta los últimos siete (7) días. Así se determina su nivel de actividad física o sedentarismo que realizan.

\section{Resultados}

Al analizar los resultados se emiten los valores de los datos analizados por estadística descriptiva. Por ejemplo, la tabla 1 determina los datos sociodemográficos de los 54 adultos mayores adscritos a un hogar geriátrico privado de la localidad de Barrios Unidos. Esta población está constituida solo por hombres, los cuales se encuentran en un rango de edad de 68.9 años, generando una edad de adultez mayor.

Asimismo, se evidencia que el peso promedio de 72.3 kilogramos (kg), con una adecuada concordancia de la estatura de 1.70 metros (m). De esta

Tabla 1. Datos sociodemográficos adultos mayores, localidad Barrios Unidos

\begin{tabular}{|c|c|c|c|c|}
\hline Sujetos & Edad & Peso & Estatura & IMC \\
\hline 54 & $68.9^{\star} \pm$ & $72.3^{\star} \pm$ & $1.70^{\star} \pm 0.07^{\star *}$ & $25.9^{\star} \pm$ \\
& $5.37^{\star *}$ & $8.94^{\star *}$ & & $4.18^{\star \star}$ \\
\hline
\end{tabular}

Fuente. *Promedio -. **Desviación Estándar. Elaboración propia.

forma, la relación del IMC determina que el valor de 25.9 se relaciona con esta población en un estado de preobesidad. Esto aumenta los factores de riesgo de sufrir una enfermedad no transmisible o un accidente cerebrovascular.

De acuerdo con el IPAQ, la actividad física moderna representa tareas que se generen entre tres (3) o más días de tareas de mínimo 20 minutos de ejecución por día. También cinco (5) o más días de caminata por un tiempo de 30 o más minutos; cinco (5) días o más de combinación de caminata y otras actividades que generen una activación de más 600 METS (Rubio, 2017).

La actividad física vigorosa representa tareas que se generan mínimo tres (3) días por semana y que generan una activación de al menos 1500 METS. Asimismo, que se implementen siete (7) días de cualquier combinación de caminata, junto con actividad física moderada o vigorosa logrando una activación de al menos 3000 METS. 
Tabla 2. Descripción de inactividad física, promedio de día

\begin{tabular}{|c|c|c|c|}
\hline Rangos & $\begin{array}{c}\text { Total } \\
\text { personas }\end{array}$ & $\begin{array}{c}\text { Total } \\
\text { minutos }\end{array}$ & $\begin{array}{c}\text { Promedio } \\
\text { de MET }\end{array}$ \\
\hline $60-65$ & 12 & 6350 & 551,428 \\
\hline $66-70$ & 24 & 14075 & 570,625 \\
\hline $71-75$ & 10 & 6510 & 705 \\
\hline $76-80$ & 8 & 4380 & 630 \\
\hline
\end{tabular}

Fuente. Elaboración propia.

La tabla 3 determina los valores en minutos y MET de los adultos mayores, generado por auto reporte o ayuda del cuidador. Para los adultos de 60 a 65 años presentan un total de 6350 minutos al día en posición de quietud, generando durante este tiempo un promedio de 551,42 MET. Para los adultos de 66 a 70 años presentan un total de 14075 minutos al día en posición de quietud, teniendo en cuenta que es el grupo más representativo. Por ello, desarrollan un promedio de 580,62 MET.

Entonces, se evidencia que entre más longevo sea el sujeto, más inactivo es, desarrollando mayores factores de riesgo.

Tabla 3. Valores en sumatoria de METS por consumo energético

\begin{tabular}{|l|c|c|c|c|c|}
\hline $\begin{array}{c}\text { Sumatoria de } \\
\text { METS en AF }\end{array}$ & $\begin{array}{c}\text { No. } \\
\text { sujetos }\end{array}$ & $\begin{array}{c}\text { Promedio } \\
\text { Edad }\end{array}$ & $\begin{array}{c}\text { Promedio } \\
\text { Peso }\end{array}$ & $\begin{array}{c}\text { Promedio } \\
\text { Talla }\end{array}$ & $\begin{array}{c}\text { Promedio } \\
\text { IMC }\end{array}$ \\
\hline 0-2000 METS & 28 & 69,8461 & 75,5384 & 1,67 & 27,21 \\
\hline 2001-4000 METS & 18 & 68,22 & 69,13 & 1,68 & 24,6 \\
\hline 4001-6000 METS & 3 & 67,66 & 76,66 & 1,62 & 29,38 \\
\hline 6001-8000 METS & 1 & 69 & 60 & 1,77 & 19,15 \\
\hline 8001-10000 METS & 1 & 66 & 74 & 1,77 & 23,62 \\
\hline 10001-12000 METS & 0 & 0 & 0 & 0 & 0 \\
\hline 12001-14000 METS & 3 & 70,33 & 66 & 1,74 & 21,85 \\
\hline
\end{tabular}

Fuente. Elaboración propia.

\section{Discusión}

De acuerdo con los hallazgos generados en este estudio, se pudo determinar que los adultos mayores presentan condiciones de riesgo al generar un estado de preobesidad con un IMC 25.9. Por ello, estudios realizados determinan que aumentar los niveles de actividad física evita los riesgos de desarrollar 
hipertensión arterial, diabetes mellitus tipo dos (2), obesidad y otras enfermedades no transmisibles (Zhang-Xu, Vivanco, Zapata, Málaga y Loza, 2011; Gómez y Melo, 2019). Por lo anterior, es necesario que se generen actividades con un mínimo de intensidad, duración y frecuencia (Pate, et al, 1995).

De igual manera, también se encontró un estudio en donde se pudo determinar que la práctica de actividad física de 20 a 60 minutos entre dos (2) a cinco (5) veces por semana. En especial, los trabajos de resistencia se podían implementar como tratamiento y prevención de la sarcopenia en adultos mayores. Así, produce un aumento de la masa y la fuerza muscular; además de un incremento en la síntesis de proteínas musculoesqueléticas y tamaño de la fibra muscular (Rubio de Peral y Gracia, 2018).

En el siguiente estudio se pudo determinar que la práctica de actividad física moderada entre 30 minutos al día, cinco (5) veces por semana, o actividad física vigorosa de 20 minutos diarios, mínimo tres (3) días a la semana. Permite tener amplios beneficios en la salud sobre adultos mayores que tengan riesgos cardiovasculares y otras enfermedades crónicas no transmisibles (Zhang-Xu, Vivanco, Zapata, Málaga y Loza, 2011).

Por otro lado, se encontró que a medida que se envejece, el nivel de actividad física disminuye. Con ello, aumentan todos los factores de riesgos asociados, los cuales directamente repercuten sobre un estado óptimo de salud. Asimismo, se encontró que las posibilidades de ser regularmente activo fueron mayores en los varones (Gómez, Duperly, Lucumí, Gámez y Vanegas, 2005).

Entonces, se puede determinar a través del siguiente estudio que de acuerdo con el rango de edad en el cual se encuentre una persona, así se ubicará en un grupo poblacional que tendrá un gasto determinado de METS. Esto permite evidenciar cuáles son las actividades que ejecutan diariamente de las cuales se obtiene el mayor gasto energético.

De esta manera, se encontró que el grupo de menos de 50 años mostró un nivel de actividad significativamente mayor, en lo correspondiente a actividades del trabajo y transporte. Al contrario de lo que sucede en las actividades relacionadas a tareas del hogar y del tiempo libre donde el gasto energético es menor que los otros grupos de edad (Serón, Muñoz y Lanas, 2010).

\section{Conclusiones}

El análisis de este estudio pudo determinar que los adultos mayores del hogar geriátrico de la localidad de Barrios Unidos, tienen factores de riesgo asociados a su salud. Su IMC determina un estado de preobesidad, sus tiempos de inactividad física diarios son considerables, especialmente para la edad de 71 a 75; aunque no es la más longeva. 
Se pudo evidenciar que los adultos mayores entre 60-65 años son el grupo que menos realiza actividad física entre los cuatro (4) grupos evaluados; a su vez, es el más joven. Con esto se puede acotar que deben realizar mayor actividad física. En el segundo grupo se encontraron a los adultos mayores de 66-70; es el grupo más grande en número de personas en él, así como el segundo en que menos realiza actividad física.

En el tercer grupo se encuentran los adultos mayores de 71-75. Se identifica que es el grupo de adultos mayores que más actividad física realiza de los cuatro (4) grupos en la tabla. Por último, el cuarto grupo son los adultos mayores entre 76-80; se ubican como el segundo grupo que mayor actividad física realiza, algo muy favorable ya que son el grupo con mayor edad. 


\section{Referencias bibliográficas}

Alonso, A., Izquierdo, M. (2003): Condición física saludable: envejecimiento y ejercicio físico (I). Selección, 12(1), 28-33.

Castellanos-Ruiz, J., Gómez-Gómez, D. E. y Guerrero-Mendieta, C. M. (2017). Condición física funcional de adultos mayores de centros día, vida, promoción y protección integral, Manizales. Hacia la Promoción de la Salud, 22(2), 84-98. https://doi. org/10.17151/hpsal.2017.22.2.7

Cruz-Jentoft, A. J., Baeyens, J. P., Bauer, J. M., Boirie, Y., Cederholm, T., Landi, F., Zamboni, M., y otros. (2010). Sarcopenia: European consensus on definition and diagnosis: Report of the European Working Group on Sarcopenia in Older People. Age and ageing, European Working Group on Sarcopenia in Older People. Age and ageing, 39(4), 412-423. https://doi.org/10.1093/ageing/ afq034

Daza-Orozco, C. y Cera-Ochoa, R. (2018). Escritura con estilo: Guía práctica para publicar científicamente. Bogotá: Editorial Fundación Universitaria San Mateo.

Gómez L, Duperly J, Lucumí D, Gámez R, Venegas A. (2005). Nivel de actividad física global en la población adulta de Bogotá (Colombia): Prevalencia y factores asociados. Gac Sanit, 19(3), 206-13.http://dx.doi. org/10.1157/13075953
Gómez, D. y Melo, L. (2019). Simulación de colchoneta que alterna puntos de apoyo para pacientes con movilidad reducida. Revista Mare Ingenii, 1(1), 49-64. Recuperado de http:// cipres.sanmateo.edu.co/index.php/ mi/article/view/210

Organización Panamericana de la Salud. (2011). Alerta epidemiológica para enfermedad de adultos mayores. Bogotá: Organización Panamericana de la Salud.

Padilla, C., Sánchez, P., y Cuevas, M. (2014). Beneficios del entrenamiento de fuerza para la prevención y tratamiento. Nutrición hospitalaria, 29(5), 979-988. http://dx.doi. org/10.3305/nh.2014.29.5.7313

Pate, R. R., Pratt, M., Blair, S. N., Haskell, W. L., Macera, C. A., Bouchard, C., Buchner, D., Ettinger, W., Heath, G. W., \& King, A. C. (1995). Physical activity and public health. A recommendation from the Centers for Disease Control and Prevention and the American College of Sports Medicine. JAMA, 273(5), 402-407. https://doi. org/10.1001/jama.273.5.402

Rodrigues, A., Hauser, E., Capeletto, E., Petreça, D. R., Alves Faleiro, D. J. y Zarpellon Mazo, G. (2019). Factors associated with low concern about falling in physically active older people. Revista Brasileira de Medicina do Esporte, 25(1), 17-24. https://doi.org/10.1590/1517869220192501189996 
Rubio del Peral, J. A., y Gracia Josa, M. S. (2018). Ejercicios de resistencia en el tratamiento y prevención de la sarcopenia en ancianos. Revisión sistemática. Gerokomos, 29(3), 133-137. Recuperado de http://scielo.isciii. es/scielo.php?script=sci_arttext\&pi$d=S 1134-928 \times 201$

Serón, P., Muñoz, S., y Llanas, F. (2010). Nivel de actividad física medida a través del cuestionario internacional de actividad física en población Chilena. Revista médica de Chile, 138(10), 12321239. https://dx.doi.org/10.4067/ S0034-98872010001100004 8000300133\&lng=es\&tlng=es

Vagetti, G. C., de Oliveira, V., Pereira, M., Pacífico, A., Alves, T., y de Campos, W. (2017). Association of body mass index with the functional fitness of elderly women attending a physical activity program. Revista Brasileira de Geriatria e Gerontologia, 20(2), 4958. http://dx.doi.org/10.1590/198122562017020.160160

Zhang-Xu, A., Vivanco, M., Zapata, F., Málaga, G. y Loza, C. (2011). Actividad física global de pacientes con factores de riesgo cardiovascular aplicando el "International Physical Activity Questionaire (IPAQ). Revista Médica Herediana, 22(3), 115-120. 